\begin{tabular}{||l|l||}
\hline Citation & $\begin{array}{l}\text { Keivan Zavari, Goele Pipeleers, Jan Swevers, 2014, } \\
\text { Gain-scheduled controller design: illustration on an overhead crane } \\
\text { IEEE Transactions on Industrial Electronics, Vol. 61, No. 7, pp. 3713 - 3718. }\end{array}$ \\
\hline Archived version & $\begin{array}{l}\text { Author manuscript: the content is identical to the content of the published paper, but without } \\
\text { the final typesetting by the publisher }\end{array}$ \\
\hline Published version & http://dx.doi.org/10.1109/TIE.2013.2270213 \\
\hline Publisher homepage & IEEE \\
\hline Author contact & $\begin{array}{l}\text { E-mail: keivan.zavari@kuleuven.be } \\
\text { Phone number: }+3216329261\end{array}$ \\
\hline IR & lirias, KULeuven link \\
\hline
\end{tabular}




\title{
Gain-scheduled controller design: illustration on an overhead crane
}

\author{
Keivan Zavari, Goele Pipeleers, and Jan Swevers,
}

\begin{abstract}
This paper extends a recently developed interpolation-based approach to design gain-scheduled controllers for linear parameter varying systems with a thorough evaluation, comprising both simulations and experiments, on an overhead crane system. In the first step of the approach, linear time-invariant controllers are designed for local working conditions of the system using a multi-objective $\mathcal{H}_{\infty}$ method. With the help of this method, the fundamental trade-off between reference tracking and disturbance rejection in the overhead crane control problem is analyzed. In the second step, a statespace interpolation method is used to calculate a gain-scheduled controller. Although this approach does not guarantee stability and performance under parameter variations, experiments on the crane setup show that these variations do not compromise the performance of the obtained controller.
\end{abstract}

Index Terms-LPV systems, $\mathcal{H}_{\infty}$ design, Gain-scheduling controller.

\section{INTRODUCTION}

$\mathbf{L}$ INEAR Parameter Varying (LPV) models describe linear system dynamics that vary as a function of time-varying parameters, called scheduling parameters. Although unknown a priori, these parameters can generally be measured or estimated in real time. To achieve satisfactory stability and performance for all possible parameter trajectories, LPV models demand so-called gain-scheduling (GS) controllers, which use the measurement/estimate of the scheduling parameters in computing the control signal [1], [2]. In the last decade, GS control has received significant practical interest and research effort. Many mechatronic systems, such as overhead cranes [3], electromagnetic actuators [4], wind energy conversion systems [5], [6] and surgical teleoperation systems [7] do exhibit LPV dynamics. In addition, LPV models also result from linearizing nonlinear system dynamics around a given statetrajectory. Hence, GS control is an effective and economical method for nonlinear control design in practice.

The GS controller design approach adopted in this paper is an interpolation based approach: First a set of local linear time-invariant (LTI) controllers are designed for different local working conditions of the LPV system, and subsequently, these LTI controllers are interpolated to obtain a GS controller as a function of the scheduling parameter. Interpolation based

Manuscript received October 1, 2012. Accepted for publication May 17, 2013.

Copyright (C) 2009 IEEE. Personal use of this material is permitted. However, permission to use this material for any other purposes must be obtained from the IEEE by sending a request to pubs-permissions@iee.org.

K. Zavari, G. Pipeleers and J. Swevers are with the Department of Mechanical Engineering, KU Leuven, Belgium, e-mail: keivan.zavari@mech.kuleuven.be. methods are generally considered practical and intuitive although contrary to the LPV GS controller design approaches (e.g. [8], [9], [10]) they cannot provide a guarantee for stability and performance.

This paper complements the work presented at the 12th International Workshop on Advanced Motion Control in Sarajevo, Bosnia [11]. As it is described in [11], our GS controller design approach uses a multi- $\mathcal{H}_{\infty}$ method to design local LTI controllers, and interpolates them into a GS controller using a recently developed LPV modeling method called state-space model interpolation of local estimates (SMILE) [12]. The present paper extends that work by a thorough validation of the approach on an overhead crane setup, which is a mechanical system with a lightly damped resonance.

The overhead crane system exhibits LPV dynamics where the cable length is the scheduling parameter. Although GS control has been applied to overhead cranes (e.g. [3], [13], [14]), these controller designs mainly focus on swing angle disturbance rejection and do not investigate the fundamental performance trade-offs between tracking and disturbance rejection. Relying on the multi- $\mathcal{H}_{\infty}$ controller design presented in [11], this paper presents a systematic and quantitative analysis of these trade-offs for the overhead crane. In addition to the valuable insight this yields in the underlying limits of the performance of the control problem, it confirms the applicability of the design approach.

The proposed control configuration is similar to the classical two degrees-of-freedom (TDOF) control [15] in the sense that it involves a two-input single-output controller. However, in our control configuration both controller inputs comprise a feedback signal, whereas in the classical TDOF control the reference command constitutes one of the control inputs. In addition, the proposed design methodology differs substantially from the classical TDOF control approach. The latter designs the two controller components sequentially and hence decouples disturbance rejection and reference tracking, whereas our approach considers both swing angle disturbance rejection and reference tracking simultaneously. Moreover, the proposed multi- $\mathcal{H}_{\infty}$ control design approach allows for a systematic analysis of the trade-off between these performance specifications.

Exploiting the SMILE interpolation does not guarantee closed-loop stability under scheduling parameter variations. In order to validate the GS controller there exist different approaches to provide a stability certificate for the closedloop system with time-varying parameters (e.g. [16], [17]). In this paper, the stability and performance of the GS controller for the overhead crane is demonstrated by experiments with 


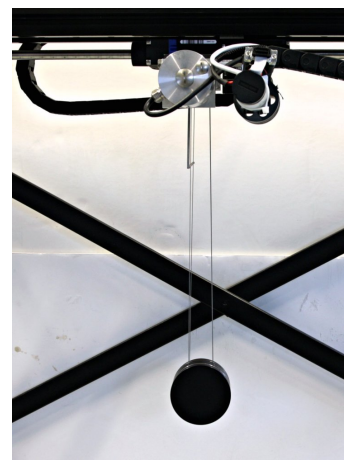

(a) Experimental test setup

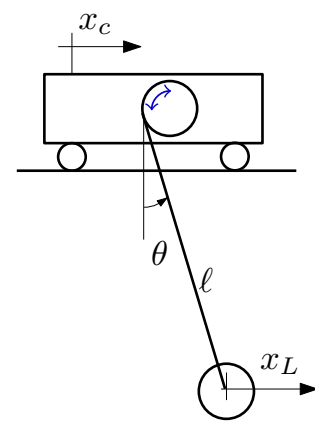

(b) Schematic diagram
Fig. 1. The overhead crane test setup and its schematic diagram.

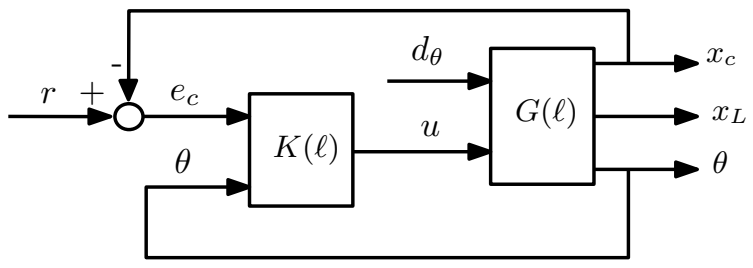

Fig. 2. Control configuration used for the overhead crane.

a time-varying scheduling parameter. This experimental validation complements the time-domain and frequency-domain simulations presented in [11].

This paper is organized as follows: Section II describes the overhead crane system test setup and the corresponding control configuration. The LTI control problem formulation together with trade-off analysis are presented in Section III The resulting GS controller and experimental validations are described and demonstrated in Section IV. Conclusions and final remarks follow in Section V

\section{EXPERIMENTAL TEST SETUP}

The overhead crane system consists of a cart to which a load is attached by a cable. Figure 1(a) shows a picture of the experimental setup, while Figure 1(b) depicts the physical system model. The cart position, load position, swing angle and cable length are indicated by $x_{c}, x_{L}, \theta$ and $\ell$, respectively.

The experimental setup is identified and controlled at a sampling frequency of $100 \mathrm{~Hz}$ using SMF Ketels EBOX (which uses EtherCAT) in combination with Orocos (Open RObot COntrol Software) [18] on a Linux operating system. The control input $u$ to the system is the speed reference for the cart's velocity control loop which ranges between 0 and $1\left[\frac{\mathrm{m}}{\mathrm{sec}}\right]$. The outputs $x_{c}, \theta$ and $\ell$ are measured by encoders (and hence known at each time instant), while the load position is calculated as $x_{L}=x_{c}+\ell \theta$. The length $\ell$ can vary between $0.35[\mathrm{~m}]$ and $0.73[\mathrm{~m}]$.

Given the high bandwidth of the cart's velocity control loop, we may assume:

$$
\frac{X_{c}(s)}{U(s)}=\frac{k}{s},
$$

where $k\left[\frac{\mathrm{m}}{\mathrm{v} \mathrm{sec}}\right]$ is a system constant relating the speed reference input $u[\mathrm{v}]$ to the cart velocity $\dot{x}_{c}\left[\frac{\mathrm{m}}{\mathrm{sec}}\right]$ and $s$ is the Laplace variable. To verify the effect of zero-order hold and possible delays caused by the hardware and software configurations in the discrete-time model of $\frac{X_{c}(z)}{U(z)}$, a frequency-domain identification is performed. The input-output data used for this identification is obtained by exciting the input $u$ by a multi-sine signal with frequency range $[0.05,5] \mathrm{Hz}$ and period $20 \mathrm{sec}$ [19]. The result is the following transfer function:

$$
\frac{X_{c}(z)}{U(z)}=\frac{0.0005}{z(z-1)}
$$

which confirms the high bandwidth of the cart's velocity control loop since no additional dynamics originating from the velocity controller is apparent in the frequency range of excitation. There is also one additional delay $\left(z^{-1}\right)$.

In order to derive the relation between $\theta$ and $x_{c}$, we use Lagrange equations for a fixed cable length $\ell$, which yields

$$
\ell^{2} \ddot{\theta}+\ell \ddot{x_{c}} \cos (\theta)+g \ell \sin (\theta)=0,
$$

where $g$ is the gravitational acceleration. For small angle $\theta$, this relation equals $\frac{\Theta(s)}{X_{c}(s)}=\frac{-s^{2}}{s^{2} \ell+g}$. This transfer function corresponds to an LTI second order system with undamped complex conjugated poles with natural frequency $f_{n}(\ell)=\frac{1}{2 \pi} \sqrt{\frac{g}{\ell}} \mathrm{Hz}$. Hence, both $f_{n}(\ell)$ and the high frequency gain of the system decrease as $\ell$ increases.

Using the bilinear transformation, the discrete-time model of $\frac{\Theta(z)}{X_{c}(z)}$ equals

$$
\frac{\Theta(z)}{X_{c}(z)}=\frac{-b(\ell)(z-1)^{2}}{z^{2}+a_{1}(\ell) z+a_{2}}
$$

where $b(\ell), a_{1}(\ell), a_{2}$ can be written as $b(\ell)=\frac{1}{\ell+2.45 \times 10^{-4}}$, $a_{1}(\ell)=\frac{-2 \ell+4.9 \times 10^{-4}}{\ell+2.45 \times 10^{-4}}$ and $a_{2}=1$.

According to (2) and (4), it is evident that the system dynamics are LPV with $\ell$ being the scheduling parameter. It is also worth mentioning that the hoisting system is velocity controlled with a bandwidth that is at least eight times higher than the resonance frequency of the system and hence, its dynamics are neglected.

This system is controlled by feeding back $\left(e_{c}, \theta\right)$ according to the configuration outlined in Figure 2. The system $G(\ell)$ and $K(\ell)$ represent the overhead crane and the controller as a function of the cable length $\ell$. Input $r$ is the reference command and the disturbance input $d_{\theta}$ imposes a swing angle disturbance such that the system response to an impulse in $d_{\theta}$ corresponds to the autonomous response from an initial swing angle $\theta_{0} \neq 0$ rad. Section III discusses the LTI control design for this system.

\section{LTI CONTROL}

The primary goal of the controller $K(\ell)$ is to make the load follow the reference command $r$ while rejecting the swing angle disturbances. In addition, the control signal should stay within the saturation limits of the actuator.

Superior load tracking requires a controller that does not excite the system's resonance and hence, has an anti-resonance at the undamped natural eigenfrequency $f_{n}(\ell)$. However, such a controller cannot compensate for load swinging due to any 
angle disturbance, as this compensation requires a harmonic control signal with frequency $f_{n}(\ell)$. Consequently, load tracking and angle disturbance rejection are conflicting performance specifications in the controller design. To analyze this trade-off using the multi- $\mathcal{H}_{\infty}$ controller design, both specifications are quantified in terms of a weighted closed-loop $\mathcal{H}_{\infty}$ norm for the shortest length $\ell=0.35[\mathrm{~m}]$. Load tracking is quantified by $\gamma_{e}=\left\|W_{e} H_{r, e_{L}}\right\|_{\infty}$ where $H_{r, e_{L}}$ denotes the closedloop transfer function from $r$ to $e_{L}=r-x_{L}$, and $W_{e}=$ $0.0126 /(z-0.999)$. This weight enforces $H_{r, e_{L}}$ to have a slope of at least $20[\mathrm{~dB} / \mathrm{dec}]$ at low frequencies, and minimizing $\gamma_{e}$ corresponds to maximizing the bandwidth. Disturbance rejection is also quantified by $\gamma_{d}=\left\|H_{d_{\theta}, \theta}\right\|_{\infty}$, such that minimizing $\gamma_{d}$ corresponds to maximizing the damping of $f_{n}(\ell)$ in $H_{d_{\theta}, \theta}$ : the closed-loop transfer function from $d_{\theta}$ to $\theta$. As a third specification in the controller design problem, a constraint on the actuator effort is added: $\left\|W_{u} H_{r, u}\right\|_{\infty}<1$, where $H_{r, u}$ denotes the closed-loop transfer function from $r$ to $u$ and $W_{u}=(z-0.999) /(20 z-9.4)$ to bound the derivative of $u$, which equals the cart acceleration. This weighting function is iteratively tuned to avoid input saturation for displacement steps of $0.1[\mathrm{~m}]$.

To conclude, analyzing the trade-off between load tracking and angle disturbance rejection under bounded actuator effort amounts to solving the following control problem for various values of the dimensionless weight $\alpha$ :

$$
\begin{array}{cc}
\underset{K \in \mathcal{S}_{6}, \gamma_{e}, \gamma_{d}}{\operatorname{minimize}} & \gamma_{e}+\alpha \gamma_{d} \\
\text { subject to } & \left\|W_{e} H_{r, e_{L}}\right\|_{\infty}<\gamma_{e} \\
& \left\|H_{d_{\theta}, \theta}\right\|_{\infty}<\gamma_{d} \\
& \left\|W_{u} H_{r, u}\right\|_{\infty}<1,
\end{array}
$$

where $\mathcal{S}_{6}$ denotes the set of internally stabilizing controllers of order six (full order controller). This problem is numerically solved using the Lyapunov shaping paradigm presented in [20]. It is well known that this method introduces a convex, yet conservative solution approach for general multi-objective control problems.

\section{A. Trade-off analysis}

To analyze the trade-off between load tracking and angle disturbance rejection, a trade-off curve between $\gamma_{e}$ and $\gamma_{d}$ is computed for the shortest cable length $\ell=0.35[\mathrm{~m}]$. This is done by solving (5) using MATLAB in combination with the SeDuMi solver [21], and the YALMIP interface [22] for different values of $\alpha$ and plotting the optimal $\gamma_{e}$ and $\gamma_{d}$ values as a function of each other. This result is shown in blackdashed line in Figure 3.

By increasing $\alpha$, the trade-off curves are traced from left to right, yielding better angle disturbance rejection, lower $\gamma_{d}$ value, at the cost of degraded load tracking, higher $\gamma_{e}$ value. The left-most point corresponds to the solution of (5) with $\alpha=0$. The corresponding optimal controller is indicated by $K_{1}$, and the optimal performance indices equal $\left(\gamma_{e, 1}, \gamma_{d, 1}\right) \approx$ $(0.32, \infty)$. The right-most point on the curve is obtained by considering $\alpha=\infty$, or equivalently, by replacing the objective (5) by $\gamma_{d}$. The corresponding controller is indicated by $K_{2}$ and its performance indices equal $\left(\gamma_{e, 2}, \gamma_{d, 2}\right)=(10.62,0.5)$.

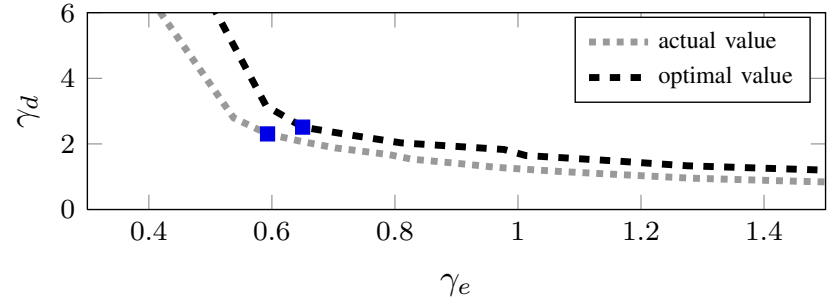

Fig. 3. Trade-off curves of tracking performance and disturbance rejection for shortest cable length $\ell=0.35 \mathrm{~m}$. The square-blue point is the tradeoff point using $K_{3}$ which provides a good compromise between conflicting performances. The axes range is restricted for visual improvements.

The steep left part of the trade-off curve indicates that starting from $K_{1}, \gamma_{d}$ can be decreased significantly with only a small increase in $\gamma_{e}$. The shallow right part, on the other hand, means that a controller can achieve significantly better load tracking performance than $K_{2}$ while having only slightly worse angle disturbance rejection. A good engineering choice would therefore be a controller that corresponds to a point on the trade-off curve with a moderate slope part, as for instance $K_{3}$, which is indicated by the blue square in Figure 3 . In order to show this moderate slope better, Figure 3 depicts a limited region of the entire trade-off curve and hence the controllers $K_{1}$ and $K_{2}$ are not visible.

To analyze the conservatism caused by the Lyapunov shaping paradigm used to solve (5), the gray dotted line in Figure 3 shows the true values of $\gamma_{e}=\left\|W_{e} H_{r, e_{L}}\right\|_{\infty}$ and $\gamma_{d}=\left\|H_{d_{\theta}, \theta}\right\|_{\infty}$ achieved by the optimized controllers. Due to the conservatism, the optimized $\gamma_{e}, \gamma_{d}$ values, obtained by solving (5) using Lyapunov shaping paradigm, provide only an upper bound to the true performance indices achieved by the optimized controller. The distance between the curves is a measure for the conservatism of the Lyapunov shaping paradigm.

In order to support the findings of the previous paragraph, the controllers $K_{1}, K_{2}$ and $K_{3}$ are further compared and implemented on the verified model of the overhead crane (2) and (4)) for the shortest length $\ell=0.35[\mathrm{~m}]$. Figure 4 compares closed-loop frequency response functions (FRFs) of $H_{r, e_{L}}, H_{r, u}$ and $H_{d_{\theta}, \theta}$, while Figure 5 shows their timedomain responses. The results for $K_{1}$, which yields the best load tracking, are shown in dashed green. The dotted purple lines correspond to $K_{2}$, which yields the best disturbance rejection, while the solid blue lines relate to $K_{3}$, which yields a good trade-off between load tracking and angle disturbance rejection. In Figure 5 the top plot shows the response to a step input of $10 \mathrm{~cm}$ in $r$ and the lower plot depicts an initial angle deviation.

Figure 4 confirms that $K_{1}$ yields the highest bandwidth for $H_{r, e_{L}}$. To achieve this superior tracking performance $K_{1}$ must not excite the system's resonance, due to which it cannot counteract the swinging initiated by an initial angle deviation. Consequently, in the FRF of $H_{d_{\theta}, \theta}$ the system's undamped resonance prevails. Figure 5 confirms the superior tracking and poor disturbance rejection performance of $K_{1}$.

The controller $K_{2}$ yields superior disturbance rejection at the cost of a very poor tracking. This is confirmed by 

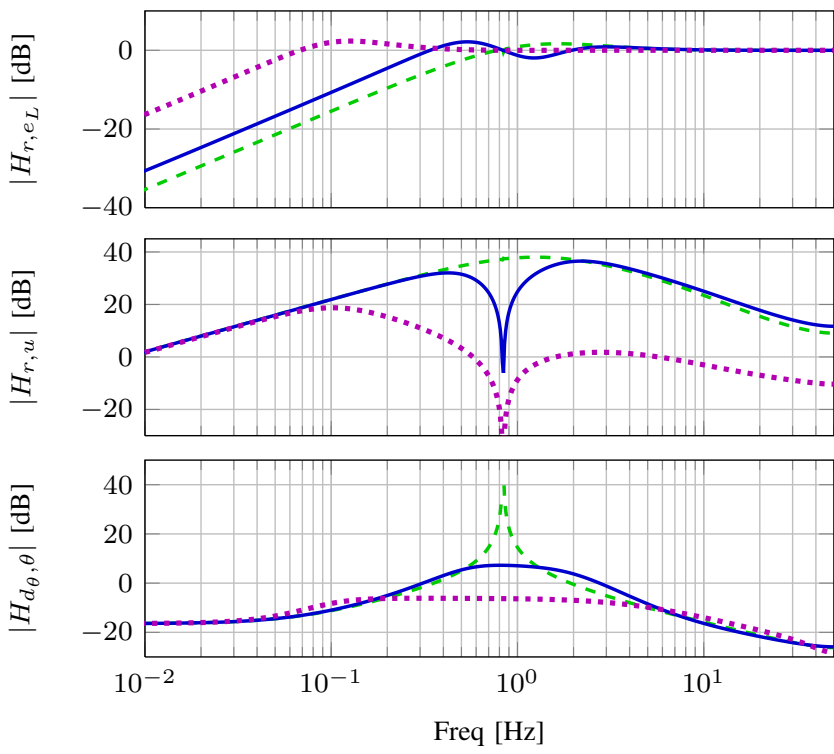

Fig. 4. Closed-loop Bode diagrams using the controller $K_{1}$ (dashed green), $K_{2}$ (dotted purple) and $K_{3}$ (solid blue).
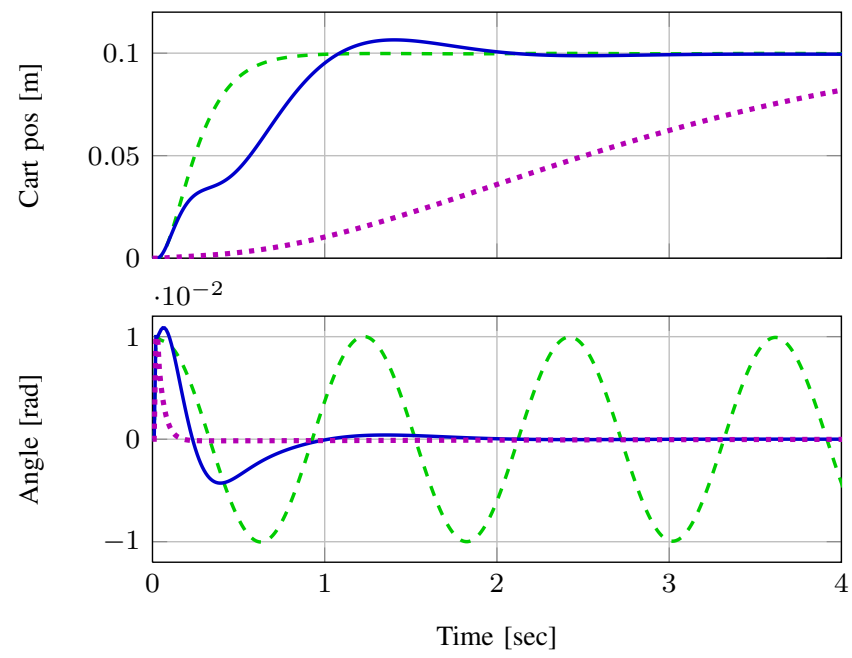

Fig. 5. Reference step response (top figure) and Swing angle disturbance response (bottom figure) using controller $K_{1}$ (dashed green), $K_{2}$ (dotted purple) and $K_{3}$ (solid blue).

frequency- and time-domain responses. Controller $K_{3}$, on the other hand, yields a good compromise between the two performance aspects. Compared to $K_{1}$ it yields a slightly lower bandwidth in $H_{r, e_{L}}$, while providing much more damping to the resonance in $H_{d_{\theta}, \theta}$. Compared to $K_{2}, K_{3}$ gives a slightly higher $\left\|H_{d_{\theta}, \theta}\right\|_{\infty}$, while providing a significantly faster time response as depicted in Figure 5.

Following the same methodology, we can design local LTI controllers for identified local models corresponding to different cable length values. Figure 6 shows the tradeoff figure for actual $\gamma$ values for cable lengths of $\ell=$ $[0.35,0.55,0.63,0.73][\mathrm{m}]$ ranging from the most dotted blue to solid red. The curves of larger $\ell$ have higher $\gamma$ values which is a consequence of the bound on the actuator effort imposed in the optimization problem (5). The bound is only active at high frequencies and the high frequency gain of system (4)

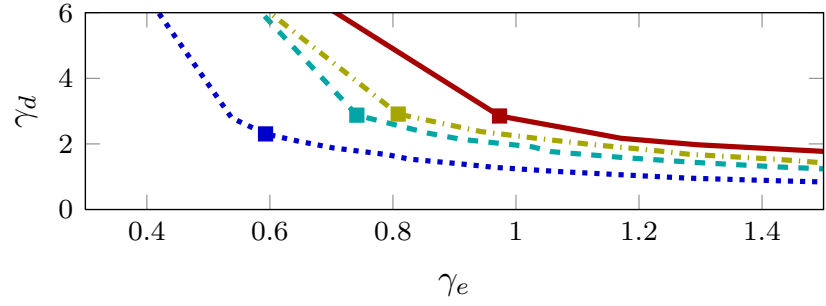

Fig. 6. Trade-off curves of tracking performance and disturbance rejection for different cable lengths $\ell=[0.35,0.55,0.63,0.73][\mathrm{m}]$ ranging from dotted blue to solid red line. The square point is the trade-off point which provides a good compromise between conflicting performances. The axes range is restricted for visual improvements.

decreases with longer cable lengths. Therefore, the bandwidth decreases for larger $\ell$ and the trade-off plot of longer cable length shifts higher. This means that for the same disturbance rejection performance the tracking gets worse for local systems corresponding to longer cable length. The local LTI controllers selected for interpolation into a GS controller using SMILE technique are indicated with squares in Figure 6 The results of this interpolation is discussed in the next section.

\section{INTERPOLATING GS CONTROLLER}

This section evaluates the GS controller on the overhead crane by interpolating the four selected LTI local controllers using the SMILE interpolation technique [12]. This technique formulates the interpolation as a linear least squares problem and calculates the state-space coefficients of the parameter dependent model as a function of the varying parameter.

The interpolation result is a polynomially parameter dependent controller of degree two. In other words, any controller in the range of variation $[0.35,0.73][\mathrm{m}]$ is calculated as:

$$
\mathcal{K}(\ell)=\left[\begin{array}{c|c}
\mathcal{A}(\ell) & \mathcal{B}(\ell) \\
\hline \mathcal{C}(\ell) & \mathcal{D}(\ell)
\end{array}\right]=\mathcal{K}_{0}+\ell \mathcal{K}_{1}+\ell^{2} \mathcal{K}_{2} .
$$

where $\mathcal{K}_{i}, i=0,1,2$ are matrix coefficients of the GS controller as the following:

$$
\mathcal{K}_{i}=\left[\begin{array}{c|c}
\mathcal{A}_{i} & \mathcal{B}_{i} \\
\hline \mathcal{C}_{i} & \mathcal{D}_{i}
\end{array}\right], \quad i=0,1,2
$$

The validation in this section comprises three parts. In the first two parts the cable length is assumed to be fixed while in the third part the validation is done for varying cable length. The first part is to compare the FRFs of the GS controller evaluated at different cable length values with the local LTI controllers in order to validate the interpolation accuracy. In the second part, the corresponding closed-loop systems using the GS controller and the local LTI controllers are compared. This is done to verify the closed-loop performance using the GS controller. The third and final part experimentally validates the GS controller for time-varying parameters.

In Figure 7 the calculated GS controller is compared to the local LTI controllers. The gray lines show the magnitude of the frequency response of the GS controller evaluated at different $\ell$ values, while the black lines changing from blue to red are the four local LTI controllers. Although a small 


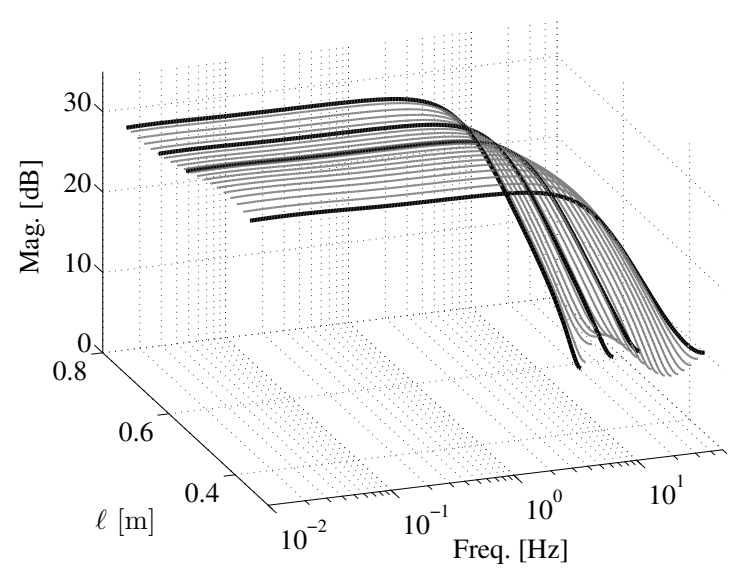

(a) Channel 1: $K_{e_{c}, u}$

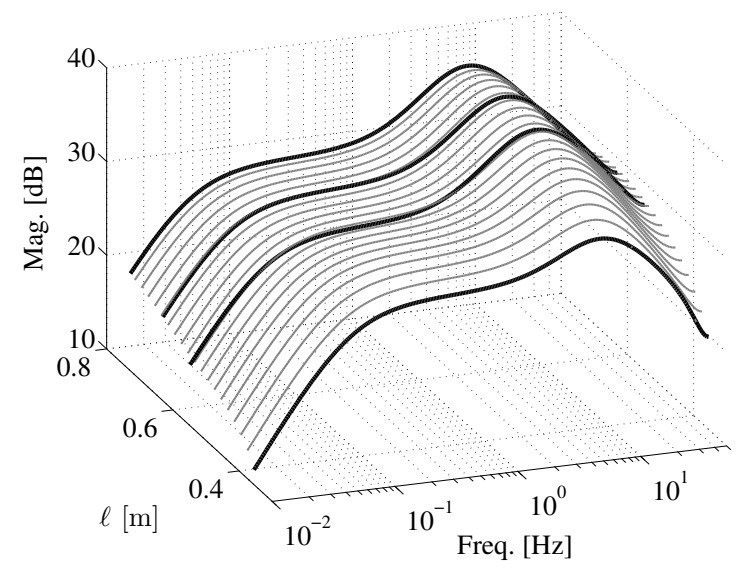

(b) Channel 2: $K_{\theta, u}$

Fig. 7. Local LTI controllers (black) and interpolating GS controller of degree two evaluated for 19 equidistant values of $\ell$ (gray).

mismatch is visible on the first channel of the controller at high frequencies, the error is not important as it only appears at frequencies much higher than the closed-loop bandwidth. Therefore comparable performance can be expected by using the GS controller.

Figure 8 illustrates the effect of interpolation on the closedloop bandwidth and the norm $\left\|H_{d_{\theta}, \theta}\right\|_{\infty}$ as a function of $\ell$. Closed-loop systems corresponding to the local LTI controllers and the GS controller evaluated at different $\ell$ values, are indicated with black-squares and green-star respectively. As it is depicted, the bandwidth decreases as the cable length increases and comparison shows that the gain-scheduled controller has a negligible lower bandwidth (less than $2 \%$ ). Figure 8 also shows that the norm value $\left\|H_{d_{\theta}, \theta}\right\|_{\infty}$ with the GS controller is slightly lower ( $6 \%$ at the most). The difference between the gain-scheduled and local LTI controller is due to the interpolation error and it is insignificant since the gainscheduled controller gives satisfactory performance.

In order to validate the GS controller with time-varying parameters, a set of experiments with varying cable length are performed. In this paper three representative results are shown: The step response with a length value varying from $\ell=0.73[\mathrm{~m}]$ to $\ell=0.35[\mathrm{~m}]$ at three different constant rates of $0.2,0.1$ and $0.05\left[\frac{\mathrm{m}}{\mathrm{sec}}\right]$. Figure 9 shows the step responses and

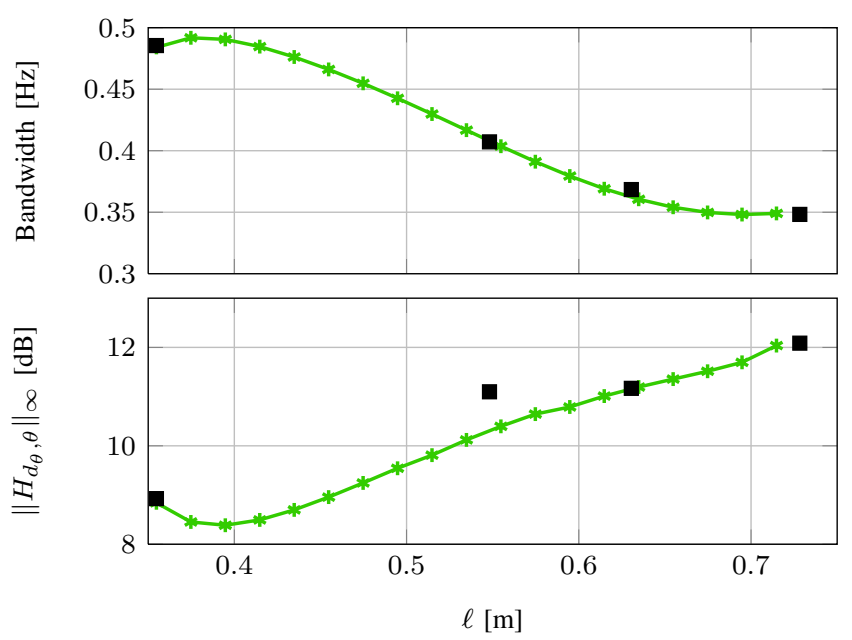

Fig. 8. Closed-loop bandwidth (top) and $\left\|H_{d_{\theta}, \theta}\right\|_{\infty}$ as a function of $\ell$ (bottom). Local LTI controllers (black squares) are compared to the gainscheduled controller (solid green) evaluated for 19 equidistant values of $\ell$.
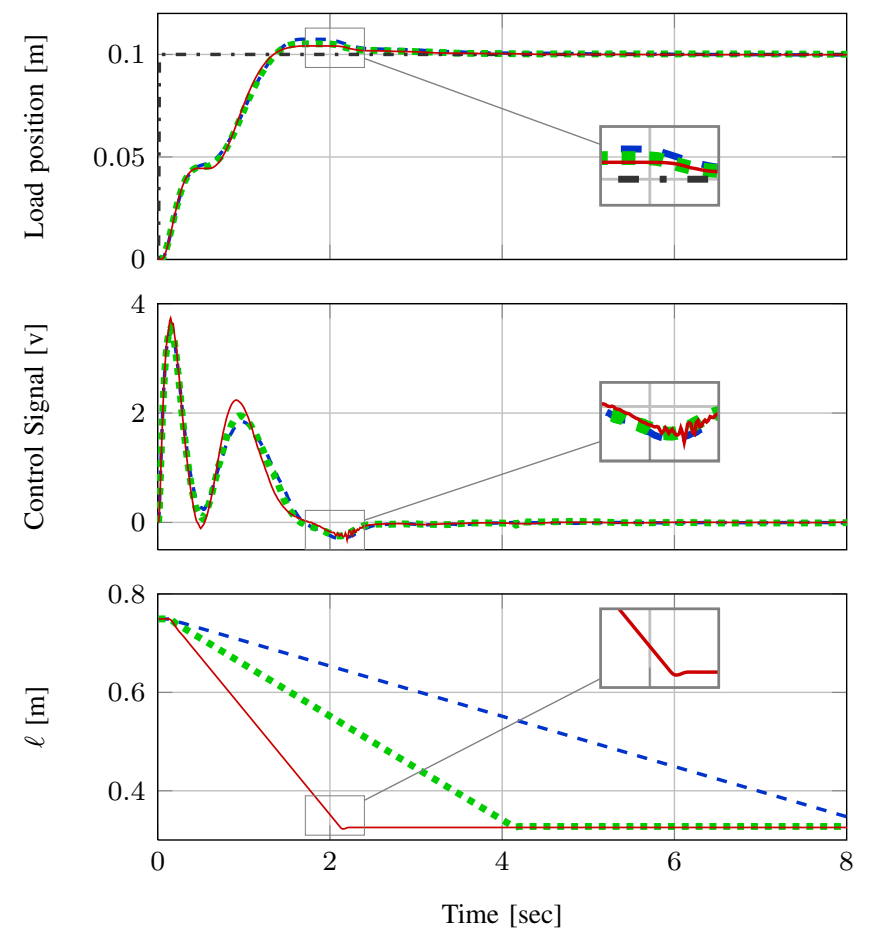

Fig. 9. Position of the load in presence of parameter variation. Fast parameter variation (solid red) compared to slower parameter variations (dotted green and dashed blue). The reference command is shown in black dash-dotted.

cable length for fast to slow variation. The fastest variation shown in solid-red line is set to $0.2\left[\frac{\mathrm{m}}{\mathrm{sec}}\right]$ such that the load is at the desired position when the step response is settled. The length change is applied at $t=0.1$ [sec] right after the reference command is set to $0.1[\mathrm{~m}]$ and as it is shown there is barely any difference in performance at fast or slow length variations. 


\section{CONCLUSION}

This paper extends the practical interpolated GS controller design approach for LPV systems presented in [11], by thoroughly investigating the approach and experimentally confirming the results on an overhead crane system with a varying cable length. In the first step, LTI control design is performed by splitting the classical $\mathcal{H}_{\infty}$ control problem into $\mathcal{H}_{\infty}$ constraints and objectives on selected closed-loop subsystems. This multi$\mathcal{H}_{\infty}$ controller design method allows us to analyze the natural trade-off between reference tracking and disturbance rejection present in the overhead crane system. The second step, is using a recently developed interpolation technique (SMILE) for LPV modeling to derive gain-scheduled controllers from local LTI controllers. The experimental results on the system reveal the value of this approach in presence of varying parameters.

\section{ACKNOWLEDGEMENTS}

This work benefits from KU Leuven-BOF PFV/10/002 Center-of-Excellence Optimization in Engineering (OPTEC), the Belgian Programme on Interuniversity Attraction Poles, initiated by the Belgian Federal Science Policy Office (DYSCO), European research project EMBOCON FP7-ICT2009-4 248940, KU Leuven's Concerted Research Action GOA/10/11, and G.0377.09 of the Research Foundation Flanders (FWO Vlaanderen). Goele Pipeleers is a Postdoctoral Fellow of the Research Foundation - Flanders.

\section{REFERENCES}

[1] D. J. Leith and W. E. Leithhead, "Survey of gain-scheduling analysis and design," International Journal of Control, vol. 73, no. 11, pp. 1001 $-1025,2000$.

[2] W. J. Rugh and J. S. Shamma, "Research on gain scheduling," Automatica, vol. 36, no. 10, pp. 1401 - 1425, 2000.

[3] G. Corriga, A. Giua, and G. Usai, "An implicit gain-scheduling controller for cranes," IEEE Transactions on Control Systems Technology, vol. 6, pp. 15-20, Jan 1998.

[4] A. Forrai, T. Ueda, and T. Yumura, "Electromagnetic actuator control: A linear parameter-varying (LPV) approach," IEEE Transactions on Industrial Electronics, vol. 54, pp. 1430 -1441, June 2007.

[5] E. Muhando, T. Senjyu, A. Uehara, and T. Funabashi, "Gain-scheduled control for wecs via LMI techniques and parametrically dependent feedback part I: Model development fundamentals," IEEE Transactions on Industrial Electronics, vol. 58, pp. 48-56, Jan. 2011.

[6] E. Muhando, T. Senjyu, A. Uehara, and T. Funabashi, "Gain-scheduled control for wecs via LMI techniques and parametrically dependent feedback part II: Controller design and implementation," IEEE Transactions on Industrial Electronics, vol. 58, pp. 57-65, Jan. 2011.

[7] J. H. Cho, H. I. Son, D. G. Lee, T. Bhattacharjee, and D. Y. Lee, "Gainscheduling control of teleoperation systems interacting with soft tissues," IEEE Transactions on Industrial Electronics, vol. 60, pp. 946 -957, Mar. 2013.

[8] A. Packard, "Gain scheduling via linear fractional transformations," Systems \& Control Letters, vol. 22, no. 2, pp. 79 - 92, 1994.

[9] P. Apkarian, P. C. Pellanda, and H. D. Tuan, "Mixed $H_{2} / H_{\infty}$ multichannel linear parameter-varying control in discrete time," Systems \& Control Letters, vol. 41, no. 5, pp. 333 - 346, 2000.

[10] C. Scherer, "LPV control and full block multipliers," Automatica, vol. 37 , no. 3 , pp. $361-375,2001$.

[11] K. Zavari, G. Pipeleers, and J. Swevers, "Interpolated gain-scheduled controllers for an over-head crane," in 12th IEEE International Workshop on Advanced Motion Control (AMC), pp. 1-6, March 2012.

[12] J. De Caigny, J. Camino, and J. Swevers, "Interpolation-based modeling of MIMO LPV systems," IEEE Transactions on Control Systems Technology, vol. 19, pp. 46 -63, Jan. 2011.

[13] J. Yi, N. Yubazaki, and K. Hirota, "Anti-swing and positioning control of overhead traveling crane," Information Sciences, vol. 155, no. 12, pp. $19-42,2003$.
[14] H. M. Omar and A. H. Nayfeh, "Gantry cranes gain scheduling feedback control with friction compensation," Journal of Sound and Vibration, vol. 281, no. 1-2, pp. $1-20,2005$.

[15] S. Skogestad and I. Postlethwaite, Multivariable Feedback Control, ch. 9. John Wiley \& Sons, second ed., 2007.

[16] F. Amato, M. Mattei, and A. Pironti, "Gain scheduled control for discrete-time systems depending on bounded rate parameters," Int. J. Robust Nonlinear Control, vol. 15, pp. 473-494, 2005.

[17] J. Daafouz and J. Bernussou, "Parameter dependent lyapunov functions for discrete time systems with time varying parametric uncertainties," Systems \& Control Letters, vol. 43, no. 5, pp. 355-359, 2001.

[18] H. Bruyninckx, "Open robot control software: the orocos project," in IEEE International Conference on Robotics and Automation, ICRA, vol. 3, pp. $2523-2528,2001$.

[19] R. Pintelon and J. Schoukens, Design of Excitation Signals, pp. 115138. John Wiley \& Sons, Inc., 2005.

[20] C. Scherer, P. Gahinet, and M. Chilali, "Multiobjective output-feedback control via LMI optimization," IEEE Transactions on Automatic Control, vol. 42, no. 7, pp. 896-911, 1997.

[21] J. F. Sturm, "Using SeDuMi 1.02, a Matlab toolbox for optimization over symmetric cones," Optimization Methods and Software, vol. 11, pp. 625-653, 1999.

[22] J. Löfberg, "YALMIP : A toolbox for modeling and optimization in MATLAB," in Proceedings of the CACSD Conference, (Taipei, Taiwan), 2004.

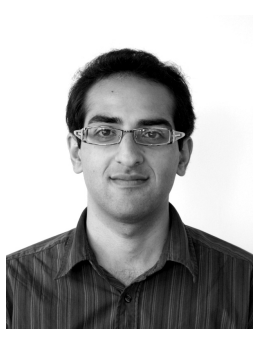

Keivan Zavari received his M.Sc. degree (with high honors) in control engineering from Tarbiat Modares University, Tehran, Iran, in 2009. He is currently working toward a Ph.D. in the Department of Mechanical Engineering, Division Production Engineering, Machine Design and Automation (PMA) of the KU Leuven. His current research is "Design of robust feedback controllers for linear parameter varying (LPV) systems". His research interests include control theory, convex optimization, and their applications to mechatronic systems.

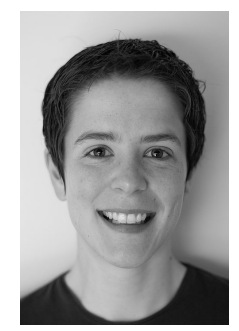

Goele Pipeleers received her M.Sc. and Ph.D. degree in mechanical engineering from the KU Leuven, Belgium, in 2004 and 2009, respectively. Currently she is a Post-doctoral Fellow of the Research Foundation-Flanders affiliated with the KU Leuven, Department of Mechanical Engineering, Division of Production Engineering, Machine Design and Automation (PMA). Her research interests include convex optimization, optimal and robust control, and their applications to mechatronic systems.

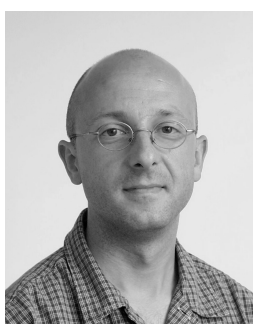

Jan Swevers received his M.Sc. degree in electrical engineering and the Ph.D. degree in mechanical engineering from the Katholieke Universiteit Leuven (KU Leuven), Belgium, in 1986 and 1992, respectively. He is since 1995 a professor in the Department of Mechanical Engineering, Division Production Engineering, Machine Design and Automation (PMA), of KU Leuven. His research interests include modeling, identification, control and optimization of mechatronics systems. http://www.mech.kuleuven.be/meco 